\title{
A Novel HBT Frequency Doubler Design for Millimeter-Wave Applications
}

\author{
Johansen, Tom Keinicke; Krozer, Viktor; Vidkjær, Jens; Djurhuus, Torsten
}

Published in:

Proceedings of the Integrated Nonlinear Microwave and Millimetre-wave Circuits (INMMiC)

Link to article, DOI:

10.1109/INMMIC.2006.283521

Publication date:

2006

Document Version

Publisher's PDF, also known as Version of record

Link back to DTU Orbit

Citation (APA):

Johansen, T. K., Krozer, V., Vidkjær, J., \& Djurhuus, T. (2006). A Novel HBT Frequency Doubler Design for Millimeter-Wave Applications. In Proceedings of the Integrated Nonlinear Microwave and Millimetre-wave Circuits (INMMiC) IEEE. https://doi.org/10.1109/INMMIC.2006.283521

\section{General rights}

Copyright and moral rights for the publications made accessible in the public portal are retained by the authors and/or other copyright owners and it is a condition of accessing publications that users recognise and abide by the legal requirements associated with these rights.

- Users may download and print one copy of any publication from the public portal for the purpose of private study or research.

- You may not further distribute the material or use it for any profit-making activity or commercial gain

- You may freely distribute the URL identifying the publication in the public portal

If you believe that this document breaches copyright please contact us providing details, and we will remove access to the work immediately and investigate your claim 


\title{
A Novel HBT Frequency Doubler Design for Millimeter-Wave Applications
}

\author{
Tom K. Johansen, Viktor Krozer, Jens Vidkjær, and Torsten Djurhuus
}

\begin{abstract}
In this paper we present a novel HBT frequency doubler design for millimeter-wave application. HBT frequency doubler theory is described which leads to accurate design equations for improved performance. The developed theory shows that a substantial improvement in the HBT frequency doubler operation can be achieved using a novel second harmonic feedback network. Preliminary measurements on a frequency doubler implemented in a high-speed InP DHBT technology demonstrates an increase in the maximum output power from $-14 \mathrm{dBm}$ to $-11.5 \mathrm{dBm}$ at an input frequency of $31.4 \mathrm{GHz}$ due to the presence of the second harmonic feedback network. We demonstrate here that the $3 \mathrm{~dB}$ improvement in performance is directly due to the feedback mechanism.
\end{abstract}

Index Terms-Coplanar waveguides (CPWs), InP HBT, millimeter-wave frequency doubler, monolithic microwave integrated circuits (MMICs).

\section{INTRODUCTION}

$\mathbf{F}$ REQUENCY multipliers are important building blocks in LO chains for radar and wireless communication systems. In these applications it is desirable to use oscillators operating at lower frequencies and with higher spectral purity. The conversion gain of passive multipliers has a fundamental limit of $1 / n^{2}$ where $n$ is the frequency multiplication factor [1]. To overcome this fundamental limit one can implement frequency multipliers with active devices. Approximate design methodologies exist for FET based multipliers [1]-[2], however these methods are not easily applicable to HBT based frequency multipliers due to the highly nonlinear base-emitter junction. A millimeter-wave SiGe HBT frequency doubler design based on reactive termination at the second harmonic at the input side and the fundamental at the output side was reported in [3]. A potential problem with this approach is that optimum reactive terminations may let the frequency multiplier operate close to the unstable region [2]. Recently, Ku- and Ka-band balanced configurations of the SiGe HBT frequency doubler with second harmonic reactive termination was reported in [4]. The balanced configuration leads to higher output power but requires a differential oscillator. In [5], a Gilbert-cell based broad-band InP DHBT frequency doubler was reported, but it suffers from excessive power consumption due to the large transistor count.

This paper reports on a HBT frequency doubler design based on a novel second harmonic feedback network. A simplified nonlinear analysis based on the harmonic-balance technique is performed to estimate the optimum excitation

The authors are with Oersted $\bullet$ DTU, Section for Electromagnetic Systems, Technical University of Denmark, 2800 Kgs. Lyngby, Denmark. (phone: +4545253770; e-mail:tkj@ oersted.dtu.dk)
0-7803-9723-1/06/\$20.00 @2006 IEEE.

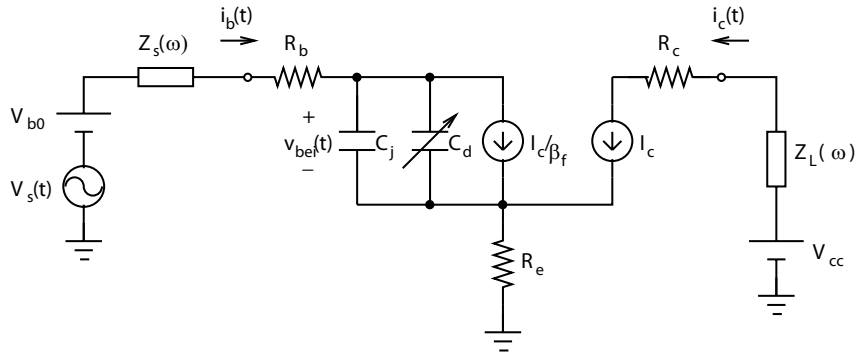

Fig. 1. Simplified nonlinear model of HBT frequency doubler.

for HBT frequency doubler performance. It turns out to be a signal containing a second harmonic component which must be generated by feeding part of the second harmonic output signal back to the input. A millimeter-wave frequency doubler design including the second harmonic feedback network has been implemented in a high-speed InP DHBT technology. Preliminary measurements verifies the benefits of the second harmonic feedback network.

\section{Frequency Doubler Theory}

A simplified nonlinear model of a HBT frequency doubler with its embedded impedances is shown in Fig. 1. The HBT is driven from a voltage source $V_{s}(t)$ with source impedance $Z_{s}(\omega)$ at its base and is terminated at the collector with a load impedance $Z_{L}(\omega)$. The source impedance is assumed to shortcircuit all frequency components except the fundamental while the load impedance short-circuits all frequency components except the second harmonic. Three nonlinearities, the base current $I_{c} / \beta_{f}$, the collector current $I_{c}$, and the base-emitter diffusion capacitance $C_{d}$, are included in the model along with fixed parasitic resistances $R_{b}, R_{e}$ and $R_{c}$. The base-emitter depletion capacitance $C_{j}$ is weakly nonlinear and can be represented by its time-averaged value. Assuming a sinusoidal voltage across the internal base-emitter junction $v_{b e i}(t)=$ $V_{b e o}+2 V_{b 1} \cos \left(\omega_{i n} t\right)$, the collector current is represented as

$$
I_{c}=I_{s} e^{\frac{V_{b e o}}{V_{t}}} e^{\frac{2 V_{b 1}}{V_{t}} \cos \left(\omega_{i n} t\right)}=I_{s} e^{\frac{V_{b e o}}{V_{t}}} \sum_{n=-\infty}^{n=\infty} \mathbf{I}_{n}(x) e^{j n \omega_{i n} t}
$$

where $\mathbf{I}_{n}$ represents modified Bessel functions of order $n$ and $x=2 V_{b 1} / V_{t}$ is the normalized drive level [6]. In Fig. 2 the normalized DC and harmonic components of the collector current are shown versus the conduction angle. For a given maximum current rating $I_{\max }$, the second harmonic current is 106 


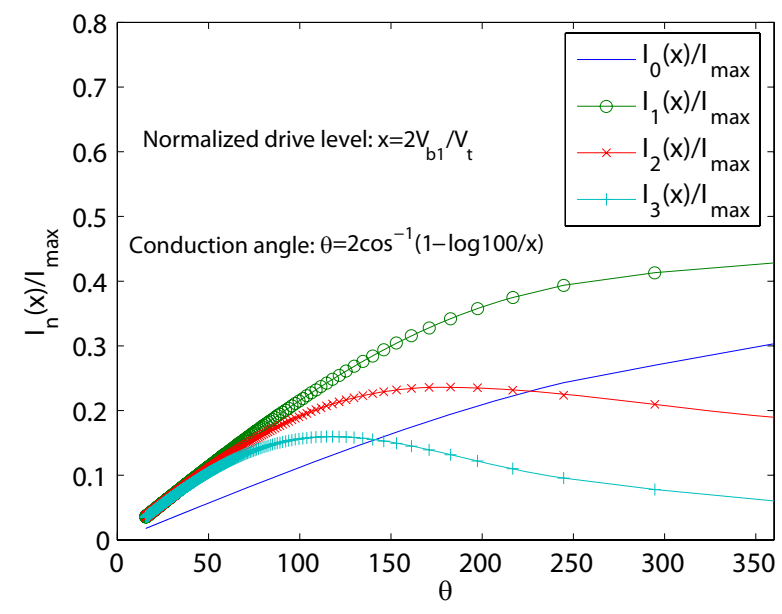

Fig. 2. Normalized DC and harmonic collector current components versus conductance angle for an internal sinusoidal base-emitter voltage.

a normalized drive level of $x=4.6^{1}$. The assumption of a sinusoidal voltage across the internal base-emitter junction is, however, generally not justified due the voltage drop caused by harmonic currents flowing through the parasitic resistances. Therefore frequency doublers based on HBT technology are not readily designable and require substantial optimization using CAD tools.

The following nonlinear analysis leads to accurate design equations for optimal HBT frequency doubler performance. The time-dependent current flowing into the base terminal can be written as

$$
i_{b}(t)=\frac{I_{c}}{\beta_{f}}+C_{j} \frac{d v_{b e i}(t)}{d t}+\tau_{f} \frac{d I_{c}}{d t}
$$

where a bias independent forward transit time $\tau_{f}$ has been assumed for simplicity. It is well-known that harmonic frequency multipliers such as a HBT frequency doubler generate only frequencies at multiple integers of the fundamental frequency $n \omega_{i n}$ [7]. Fourier transforming (2) therefore gives

$$
\begin{array}{r}
\sum_{n=-\infty}^{n=\infty} I_{b n} e^{j n \omega_{i n} t}=\sum_{n=-\infty}^{n=\infty} j n \omega_{i n} C_{j} V_{b n} e^{j n \omega_{i n} t} \\
+\sum_{n=-\infty}^{n=\infty}\left(1 / \beta_{f}+j n \omega_{i n} \tau_{f}\right) I_{c n} e^{j n \omega_{i n} t}
\end{array}
$$

where $I_{b n}, V_{b n}$, and $I_{c n}$ are Fourier coefficients at the different harmonics. The Fourier coefficients $I_{c n}$ of the collector current are expressed in terms of the Fourier coefficients of the baseemitter voltage $V_{b n}$ as follows

$$
I_{c n}=\frac{1}{T} \int_{0}^{T} I_{s} e^{\frac{\sum_{n=-\infty}^{n=\infty} V_{b n} e^{j n \omega_{i n} t}}{V_{t}}} e^{-j n \omega_{i n} t} d t
$$

where $T=2 \pi / \omega_{i n}$. Only in the case of a sinusoidal voltage across the internal base-emitter junction these are expressed in terms of modified Bessel functions as shown in (1). Applying

\footnotetext{
${ }^{1}$ Defining the conduction angle as $\theta=2 \cos ^{-1}(1-\log 100 / x)$.
}

the harmonic-balance technique yields the frequency domain representation:

$$
I_{b n}=j n \omega_{i n} C_{j} V_{b n}+\left(1 / \beta_{f}+j n \omega_{i n} \tau_{f}\right) I_{c n} .
$$

Writing Kirchoff's voltage law for the input loop in Fig. 1 and Fourier transforming gives the harmonic balance relation between voltage components $V_{s n}$ and $V_{b n}$ for the external excitation and the internal base-emitter junction, respectively,

$$
\begin{aligned}
V_{s n} & =\left[1+j n \omega_{i n} C_{j}\left(\left(Z_{s}\left(\left(n \omega_{i n}\right)+R_{b}+R_{e}\right)\right] V_{b n}\right.\right. \\
& +\left[\left(1 / \beta_{f}+j n \omega_{i n} \tau_{f}\right)\left(Z_{s}\left(n \omega_{i n}\right)+R_{b}+R_{e}\right)+R_{e}\right] \\
& \times \frac{1}{T} \int_{0}^{T} I_{s} e^{\frac{\sum_{n=-\infty}^{n=\infty} V_{b n} e^{j n \omega_{i n} t}}{V_{t}}} e^{-j n \omega_{i n} t} d t
\end{aligned}
$$

where the integral in the brackets represents the Fourier coefficients of the collector current subject to a non-sinusoidal voltage $v_{b e i}(t)$ across the internal base-emitter junction. Assuming a purely sinusoidal excitation at the internal baseemitter junction and using (6) allows for

An improved excitation can be analytically obtained from (6) if a purely sinusoidal excitation is assumed at the internal base-emitter junction and utilizing modified Bessel functions shown in (1). The external base bias voltage $V_{b o}$ is also calculated to ensure that the maximum current rating $I_{\max }$ is not exceeded. Forcing a sinusoidal base-emitter voltage across the internal junction requires however an external excitation having second and higher even harmonic components. The input impedance at the fundamental frequency is calculated as

$$
Z_{i n}\left(\omega_{i n}\right)=\frac{V_{s 1}}{I_{b 1}}-Z_{s}\left(\omega_{i n}\right)
$$

where $V_{s 1}$ and $I_{b 1}$ are the fundamental frequency components of the external excitation and the base current, respectively. The interaction between the second harmonic load, the optimal external excitation, and the input impedance can not be neglected at millimeter-wave frequencies, owing to the base-collector capacitance of the HBT. Therefore, the HBT frequency doubler theory has to be extended to include the effect of the base-collector capacitance. The analysis including the base-collector capacitance is tedious and the derivation will be shown elsewhere. A similar equation for the base current as (5) including the base-collector capacitance contribution is derived as

$$
\begin{aligned}
I_{b n}=\quad & j n \omega_{i n} C_{j} V_{b n}+\left(1 / \beta_{f}+j n \omega_{i n} \tau_{f}\right) I_{c n}+ \\
& j n \omega_{i n} C_{b c}\left(1-X_{c j c}\right)\left(V_{s n}^{\prime}-V_{c i n}\right)+ \\
& j n \omega_{i n} X_{c j c} C_{b c}\left(V_{b i n}-V_{c i n}\right)
\end{aligned}
$$

where $V_{b i n}$ and $V_{c i n}$ are the modified internal base and collector voltages, respectively.

\section{FReQuency Doubler Design}

The frequency doubler theory is now used in the design of an improved frequency doubler. The electrical parameters of the nonlinear HBT model are extracted from DC and smallsignal S-parameters measurements using standard methods, e.g. [8]. Alternatively, the electrical parameters can be deter107 
TABLE I

ELECTRICAL PARAMETERS FOR THE NONLINEAR INP DHBT MODEL

$$
\left(A_{e}=10 x 2.0 \mu m^{2}\right) \text {. }
$$

\begin{tabular}{|c|c|c|c|}
\hline Parameter & Value & Parameter & Value \\
\hline$I_{s}[\mathrm{fA}]$ & 4.5 & $R_{c}[\Omega]$ & 9.9 \\
\hline$N_{f}$ & 1.13 & $\tau_{f}[p S]$ & 1.7 \\
\hline$\beta_{f}$ & 28.1 & $C_{j}[\mathrm{fF}]$ & 55.0 \\
\hline$R_{b}[\Omega]$ & 23.0 & $C_{b c}[\mathrm{fF}]$ & 20.2 \\
\hline$R_{e}[\Omega]$ & 3.0 & $X_{c j c}$ & 0.69 \\
\hline
\end{tabular}

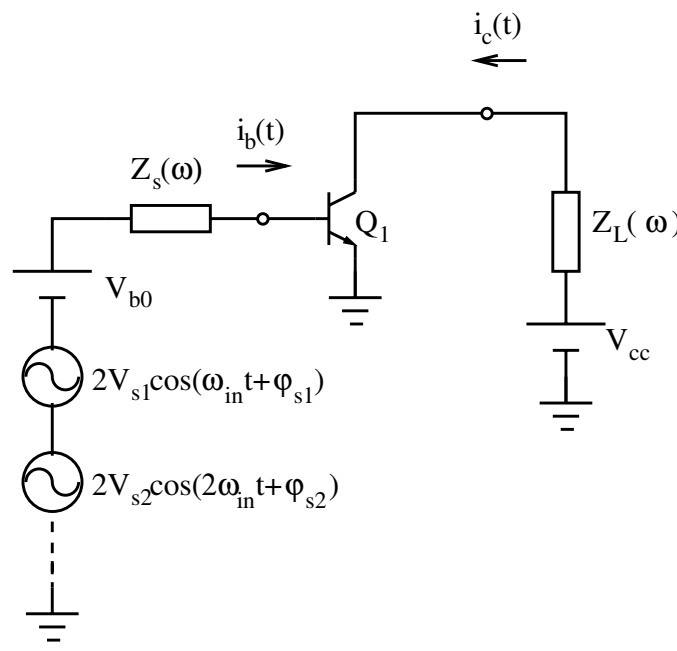

Fig. 3. Improved excitation scheme for the HBT frequency doubler.

TABLE II

CALCULATED HARMONIC VOLTAGE COMPONENTS AND INPUT IMPEDANCE FOR AN INP DHBT FREQUENCY DOUBLER $\left(f_{\text {in }}=41.75 \mathrm{GHz}\right)$.

\begin{tabular}{|c|c|c|c|c|}
\hline$V_{b o}[\mathrm{~V}]$ & $2 V_{s 1} \angle \phi_{s 1}[\mathrm{~V}]$ & $2 V_{s 2} \angle \phi_{s 2}[\mathrm{~V}]$ & $\ldots$ & $Z_{i n}[\Omega]$ \\
\hline 0.727 & $0.216 \angle 42.3^{0}$ & $0.194 \angle 69.0^{0}$ & $\ldots$ & $24.0-j 26.3$ \\
\hline
\end{tabular}

available. The electrical parameters of the nonlinear model for the InP DHBTs used in this work are given in Table. I. The harmonic voltage components and input impedance required for the sinusoidal voltage across the internal base-emitter junction are determined from (6) and (7), extended to include the base-collector capacitance. Fig. 3 illustrates an improved excitation scheme for the HBT frequency doubler. Notice that both the magnitude and phase relation are important for appropriate harmonic voltage component generation across the internal base-emitter junction. Table. II gives the calculated harmonic voltage components and input impedance required for the design of an InP DHBT frequency doubler operating at $f_{i n}=41.75 \mathrm{GHz}$ and having an optimal normalized sinusoidal drive level of $x=4.6$. The base bias voltage is calculated to keep the peak current below $I_{\max }=20 \mathrm{~mA}$. The collector current waveform obtained using this excitation is shown in Fig. 4. A strong second harmonic component is evident in the output collector current waveform. Furthermore, it is observed that the peak value of the collector current is kept slightly below $I_{\max }$. The excellent agreement with

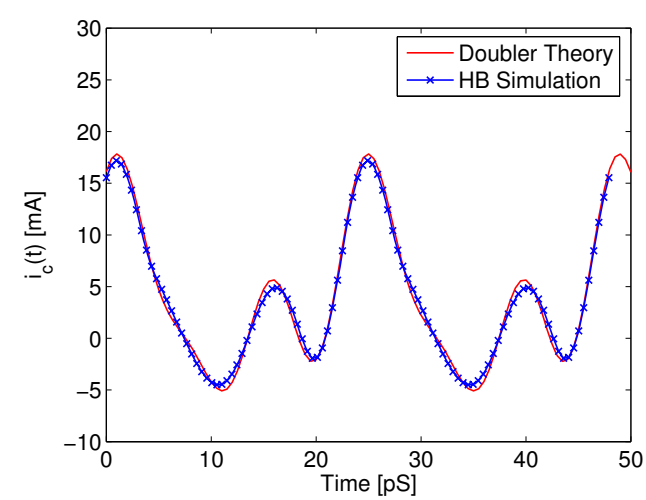

Fig. 4. Collector current waveform calculated with the frequency doubler theory (-) and ADS harmonic balance simulation (-x-).

$\mathrm{ADS}^{2}$ harmonic balance simulation verifies that a sinusoidal drive level has indeed been obtained across the internal baseemitter junction. In other words, design equations for improved frequency doubler performance have been obtained.

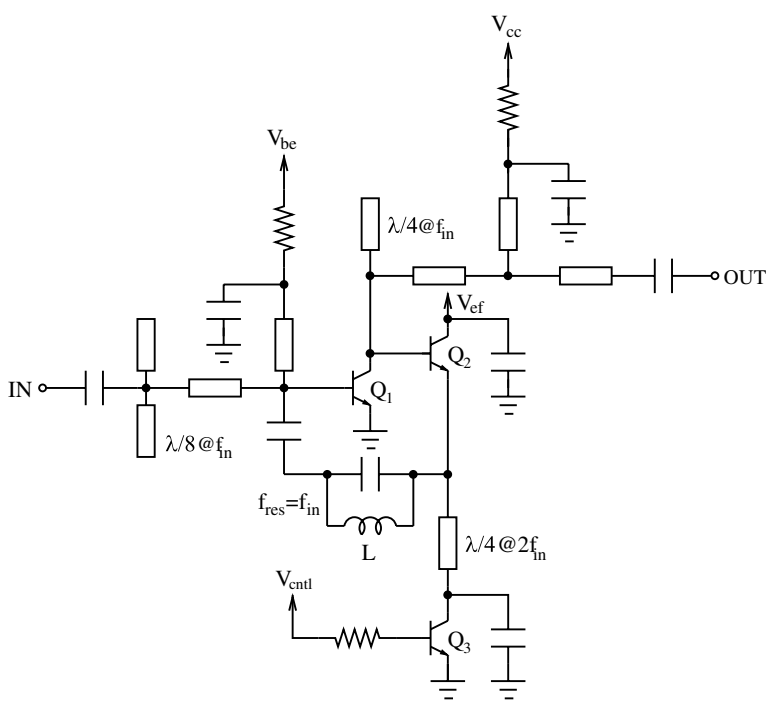

Fig. 5. Schematic of frequency doubler including second harmonic feedback network.

The second harmonic voltage needed for the excitation determined from design equations must be generated by feeding part of the output signal back to the input. The influence from higher harmonics are not as important and will therefore be ignored. The frequency doubler including the novel second harmonic feedback network is shown in Fig. 5. The parallel tuned circuit assures maximum isolation between input and output of $Q_{1}$ for the fundamental frequency signal and presents a pure reactance at the second harmonic. This reactance forms a voltage divider together with the input matching network at the second harmonic. The purpose of $Q_{3}$ is to control the amount of feedback. If the control voltage $V_{\text {cntl }}$ is set to zero the second harmonic feedback network is turned off. 
At the second harmonic frequency the input matching network presents a pure reactance to the base of $Q_{1}$, which is assured by splitting the shunt stub at the input into two parts as shown in Fig. 5. If one of these is $\lambda / 8$ long at the fundamental frequency it turns into a short circuit at the second harmonic and is transformed as a pure reactance along the transmission line towards the input of $Q_{1}$. This susceptance adds to the admittance of the short-circuited shunt stub feeding base bias to $Q_{1}$. Stable operation of the HBT frequency doubler is assured by short-circuiting the fundamental frequency component at the output through a $\lambda / 4$ long open stub.

\section{EXPERIMENTAL RESULTS}

The frequency doubler was fabricated in a high-speed InP/InGaAs DHBT circuit technology developed at the Alcatel-Thales III-V laboratory. The transistors in this technology exhibit $180 / 210 \mathrm{GHz} f_{t} / f_{\max }$, breakdown voltage $B V_{\text {ceo }}>7 \mathrm{~V}$ and yield better than $99 \%$ [9]. The technology also offers three Au/Ti metallization layers, Ti resistors, and SiN metal-insulator-metal (MIM) capacitors. The microphotograph of the fabricated frequency doubler is shown in Fig. 6. The chip size with pads is $1.4 \times 1.6 \mathrm{~mm}^{2}$. Coplanar waveguide $(\mathrm{CPW})$ transmission lines are implemented using ADS momentum or HFSS ${ }^{3}$.

Preliminary on-wafer measurements have been performed on the fabricated circuit. The measurement result given here correspond to a substrate height of $350 \mu \mathrm{m}$ instead of 120 $\mu \mathrm{m}$ assumed in the design of the frequency doubler. This has little influence on the performance of the CPW structures, however, the small interconnects around the second harmonic feedback network will behave differently, leading to an overall low conversion gain, and downward shift in frequency. Despite of these complications, the benefits of the second harmonic feedback network is evident in the output power versus control voltage shown in Fig. 7. In this figure a rise in output power of $2.5 \mathrm{~dB}$ is observed when the second harmonic feedback network is active. For a properly operating HBT frequency

${ }^{3}$ HFSS v.9, Ansoft Corp., San Jose, CA

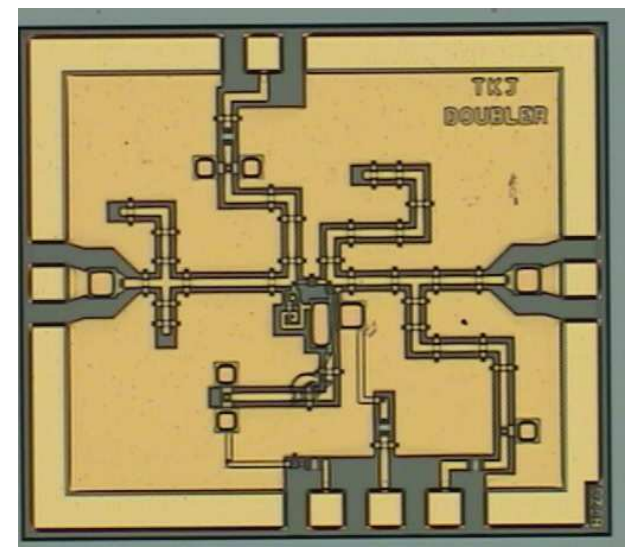

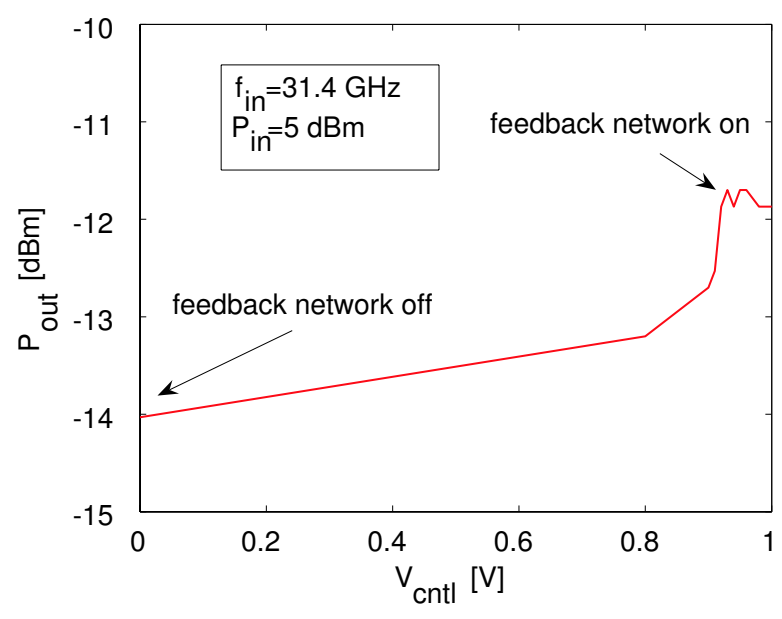

Fig. 7. Second harmonic output power versus control voltage $\left(V_{c n t l}\right)$.

doubler the expected increase in output power is more than 6 $\mathrm{dB}$.

\section{CONCLUSION}

A nonlinear theory have been developed using the harmonic balance principle which leads to accurate design equations for HBT frequency doublers. Due to the strongly nonlinear baseemitter junction optimal performance for a HBT frequency doubler requires a second harmonic voltage components within its excitation. This second harmonic components must practically be generated by feedback. A HBT frequency doubler including a novel second harmonic feedback network was purposed. The experimental results demonstrated a $2.5 \mathrm{~dB}$ increase in second harmonic output power when the second harmonic feedback network was active.

\section{REFERENCES}

[1] F. Giannini and G. Leuzzi. Nonlinear Microwave Circuit Design. John Wiley \& Sons, 1. edition, 2004.

[2] S. A. Maas. Nonlinear Microwave and RF Circuits. Artech House, 2. edition, 2003.

[3] S. P. O. Bruce et al. Design and Realization of a Millimeter-Wave Si/SiGe HBT Frequency Multiplier. IEEE Trans. Microwave Theory and Tech., 46(5):695-700, may 1998.

[4] J. J. Hung, T. M. Hancock, and G. M. Rebeiz. High-Power HighEfficiency SiGe Ku- and Ka-Band Balanced Frequency Doublers. IEEE Trans. Microwave Theory and Tech., 53(2):754-761, feb. 2005.

[5] V. Puyal et al. A broad-band active frequency doubler operating up to $120 \mathrm{GHz}$. In 13th GAAS symposium, pages 557-560, 2005.

[6] G. Boeck, K. Schmidt, and M. Tempel. Planar Microwave Mixers for Wireless and Sensor Applications. In Proc. SBMO / IEEE MTT-S IMOC 2001, pages 233-243, 2001.

[7] R. E. Lipsey and S. H. Jones. Accurate Design Equations for 50-600 $\mathrm{GHz}$ GaAs Schotky Diode Varactor Frequency Doublers. IEEE Trans. Microwave Theory and Tech., 45(9):1876-1882, sept. 1998.

[8] M. Reisch. High-Frequency Bipolar Transistors. Springer, 1. edition, 2003.

[9] S. Blayac et al. MSI InP/InGaAs DHBT technology: beyond $40 \mathrm{Gbit} / \mathrm{s}$ circuits. In 14th Indium Phosphide and Related Materials Conference, pages 51-54, 2002.

Fig. 6. Microphotograph of the feedback frequency doubler. $\left(1.4 x 1.6 \mathrm{~mm}^{2}\right.$ with pads). 